\title{
Fractal approach to description of the auroral structure
}

\author{
B. V. Kozelov \\ Polar Geophysical Institute, Apatity, Murmansk Region, 184209 Russia \\ Received: 27 September 2002 - Revised: 23 April 2003 - Accepted: 25 April 2003
}

\begin{abstract}
During the last two decades the fractal geometry has become a powerful approach to different physical problems. It is also found to be useful in image processing applications. A numerical quantity that characterizes the auroral structure would be important for auroral investigations. We try to obtain the quantity on the basis of the box-counting dimension of the line of equal intensity. In this paper we present results of some tests of our procedure by simulated images. The possibilities that the approach gives us for analysis of the auroral dynamics are discussed. The auroral dynamics during several typical auroral events are considered.
\end{abstract}

Key words. Ionosphere (auroral ionosphere) - Magnetospheric physics (magnetosphere-ionosphere interactions) Space plasma physics (nonlinear phenomena)

\section{Introduction}

Ground-based observations of the polar aurora have very long traditions in geophysics. Small-scale auroral structures and distortions have been photographed, and their generation mechanisms have been discussed since the early 1950s. Now it is known that the aurora variety is a result of numerous processes occurring in the different regions of the magnetosphere-ionosphere system: energization due to parallel electric fields, Kelvin-Helmholtz instability, wave-particle interactions, current sheet pitch-angle scattering, field-line resonances, etc. (see, for example, reviews in Lyons et al., 1999; Partamies, 2001).

Investigation of these processes usually requires a separation of temporal and spatial variations. Ground-based television (TV) observations give the data which contain twodimensional spatial and very good temporal information. However, now the images themselves are mainly used only

Correspondence to: B. V. Kozelov

(kozelov@pgi.kolasc.net.ru) as illustrations. Other methods of data presentation (for example keograms) contain only a small part of important information that is available in TV data. It would be useful to construct a numerical quantity to characterize auroral structures more completely. The fractal geometry is a powerful approach to the problem. There are many interesting results obtained by fractal geometry in other geophysical applications (for example, application to cloud structures, to geological structures, etc.). There are some reasons for application of the similar approach to aurora observations.

Even early investigators note the fact that the fine distortions of the auroral structure have some kind of selfsimilarity. In 1970, Hallinan and Davis (1970) categorized morphologically the fine vortical structures in three different groups, according to their size, lifetime, rotational sense, and reversibility. These groups were named folds, curls, and spirals. Often the vortical structures make the periodical systems vortical streets. However, the vortical structures from different groups can appear simultaneously. Modern data of ground-based and satellite observations show the parallels between characteristics of the vortical structures in a wide range from hundreds of meters to hundreds of kilometers (Trondsen and Cogger, 1998). Self-similarities have also been obtained (Lui et al., 2000) for characteristics of the auroral blobs by satellite observations.

In our previous paper (Kozelov, 1997) we presented the fractal approach for the description of the auroral images. The approach is based on the calculation of the spectrum of the box-counting dimension of isolines on auroral images. Some examples of the approach application to TV data of ground-based auroral observations was shown in Kozelov (2000). Here we have the purpose to present more detailed substantiation of the approach and to illustrate its application to analysis of auroral structures. After definition of the image processing algorithm we discuss some substantiations and provide the tests of this procedure by simulated images. Then we consider the possibilities which the approach gives us for analysis of auroral dynamics. We follow the auroral dynamics by the evolution of the isoline dimension spectrum 


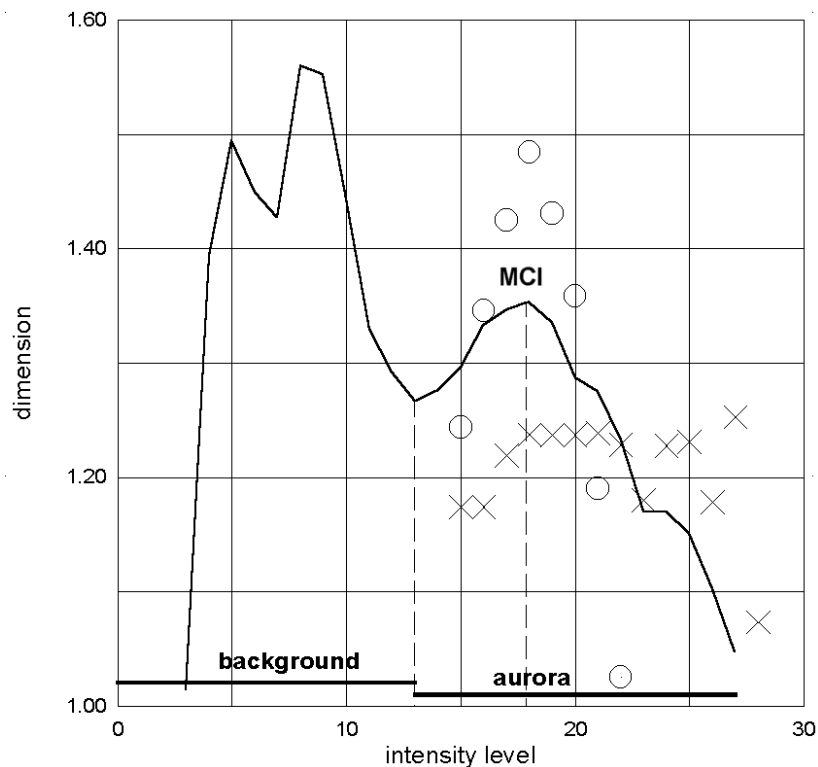

Fig. 1. Box-counting dimension of isoline as a function of intensity level: solid line - for full image; crosses - for linear arc; circles for diffuse band.

during several typical auroral events.

\section{Definition of the isoline dimension spectrum (IDS)}

To characterize the structure of the auroral image we use the fractal dimension spectrum of lines of equal intensity (Kozelov, 1997, 2000). The calculation procedure used is based on the box-counting method (Feder, 1988) and contains the following steps:

1. The isoline of equal intensity $L(I)$ has been obtained for each level of intensity $I$ in a digitized TV image;

2. Using pixels as a mesh, the number $N(\delta, I)$ of boxes of side $\delta\left(\delta=2^{i}, i=0,1, \ldots 7\right)$ that overlap the isoline $L(I)$ have been counted;

3. The dimension $D(I)$ of isoline $L(I)$ is the logarithmic rate at which $N(\delta, I)$ increases as $\delta$ decrease, and it has been estimated by the gradient of the graph of $\log N(\delta, I)$ against $-\log \delta$ for each $I$.

The box-counting dimension obtained in the calculation procedure is the estimation of fractal dimension (Falconer, 1995). However, the range of scales available in the TV image is not large (TV image in VHS system contains of 230-240 lines, real spatial scales depend on optical lens used and the distance to the object); therefore, we mean only prefractal structure, not the fractal one, which suggests a limit for $\delta \rightarrow 0$. In any case, the number of mesh boxes of side $\delta$ that intersect an isoline is an indication of how spread out or irregular the line is when examined at scale $\delta$. The dimension reflects how rapidly the irregularities develop as $\delta$ decreases. Theoretically, the dimension of the subset of a plane may have a value in the range from 0 (dimension of disconnected set of points) to 2 (dimension of plane figures). The dimension of a smooth line is equal to 1 .

\section{Example of image analysis}

An example of processing of one TV frame using the above method is shown in Fig. 1. The dependence $D(I)$ for the frame is typical, and therefore we shall consider it in detail. The frame contains an area of auroral emission consisting of a quiet linear arc and a diffuse band. The lines of equal intensities (isolines) for three levels of intensity are shown in Fig. 2. Below the level $I=9$ in the emission area in the frame there is only a small-sized structure associated with the background (background sky glow and stars) and the circuit noise. One can see from the figure for $I=8$, that the isoline in this area consists of a set of small-sized units, and therefore the isoline dimension depends mainly on how densely these units cover the plane, i.e. on their quantity. Further, as $I$ is higher than level 9 , the auroral structure is localized from the noise. This area has rather smooth boundaries, and therefore in dimension spectrum the minimum is derived at $I=13$. The isoline for $I=13$ separates the area filled by background noise from the area of auroral forms.

For intensities appropriate to auroral forms, the most essential is the presence of the local maximum on $D(I)$, in this case at the intensity $I=18$. Let us name this intensity as an intensity of the Most Complex Isoline (MCI). As we can see further, the $D(I)$ may have several of such local maxima when there are several structures of different intensity. In the considered case the auroral emission consists of two different forms, a linear arc and a diffuse band, having different dependence $D(I)$. However, these forms have the same intensity, and therefore only one common maximum is shaped. Above the level $I=15$, at which the forms are divided, it is possible to calculate the dependence $D(I)$ for each form separately. The obtained dependences are shown in Fig. 1, together with the integral dependence. One can see that the local maximum at $I \sim 18$ is caused by the influence of only the diffuse band $\left(D_{\max } \sim 1.5\right)$, while the dependence $D(I)$ for the arc in a broad region of intensities (from 17 up to 25) is practically constant $(\sim 1.22-1.24)$.

As we already noted above, the fractal (box) dimension reflects how rapidly the isoline length (or "distorsion") increases as spatial scale decreases. But what dimension do we obtain, if in the image there are two (or more) different structures? It is possible to show (Falconer, 1995), that the fractal dimension of association of several (not intersected) fractal sets is equal to the maximum of the dimensions of these sets. This is fair at $\delta \rightarrow 0$. However, for the boxcounting dimension calculated in the limited range of scales the following rule is valid. 

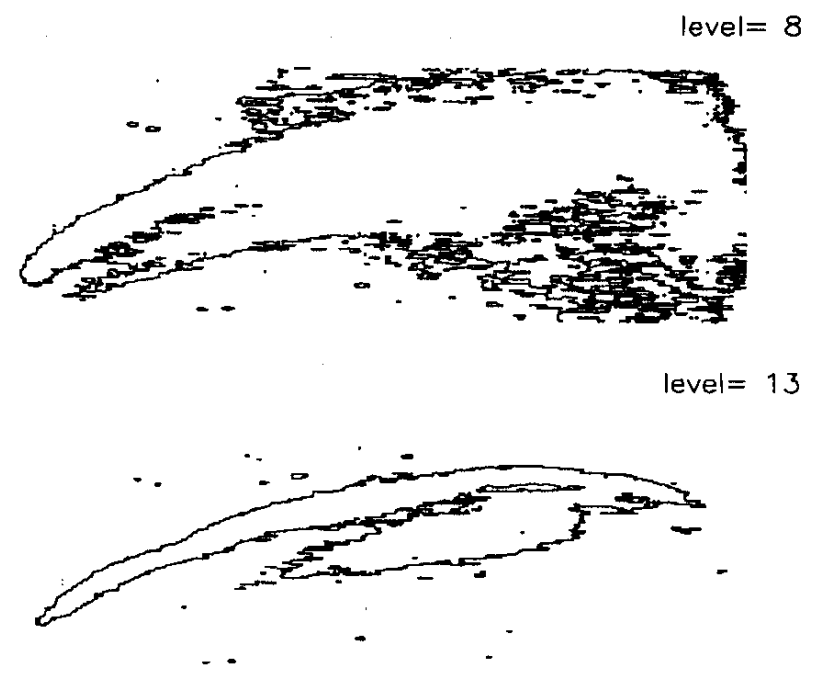

level $=18$

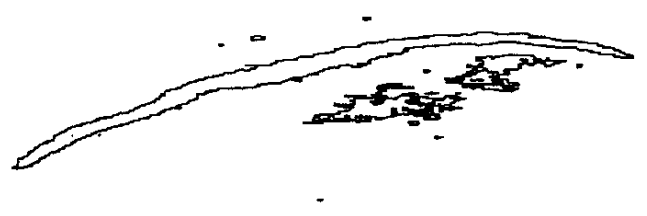

Fig. 2. Isolines for different intensity levels.

\subsection{Proposition}

Let $S_{1}$ and $S_{2}$ be the unintersected fractal sets, and $D_{1}$ and $D_{2}$-those box-counting dimensions, respectively. If for a box scale of $\delta>0$ the number of boxes that overlap the sets are $N_{1}(\delta)$ and $N_{2}(\delta)$, respectively, then the estimation of the box-counting dimension of the $S_{1} \smile S_{2}$ set may be obtained as:

$D_{1+2}(\delta) \approx \frac{N_{1}(\delta) D_{1}+N_{2}(\delta) D_{2}}{N_{1}(\delta)+N_{2}(\delta)}$.

\subsection{Proof}

For $d \delta \ll \delta$ using the definition of $D_{1}$ we can write:

$\log N_{1}(\delta+d \delta)=\log N_{1}(\delta)-D_{1} \log \left(\frac{\delta+d \delta}{\delta}\right)$.

Therefore:

$N_{1}(\delta+d \delta)=N_{1}(\delta)\left(\frac{\delta+d \delta}{\delta}\right)^{-D_{1}} \approx N_{1}(\delta)\left(1-D_{1} \frac{d \delta}{\delta}\right)$

Similarly, by definitions of $D_{2}$ and $D_{1+2}$ :

$N_{2}(\delta+D \delta) \approx N_{2}(\delta)\left(1-D_{2} \frac{\delta+d \delta}{\delta}\right)$

$N_{1+2}(\delta+d \delta) \approx N_{1+2}(\delta)\left(1-D_{1+2} \frac{\delta+d \delta}{\delta}\right)$.

For unintersected sets $N_{1+2}(\delta)=N_{1}(\delta)+N_{2}(\delta)$, therefore from Eq. (4) we obtain:

$N_{1}(\delta+d \delta)+N_{2}(\delta+d \delta) \approx\left(N_{1}(\delta)+N_{2}(\delta)\right)\left(1-D_{1+2} \frac{d \delta}{\delta}\right)$.

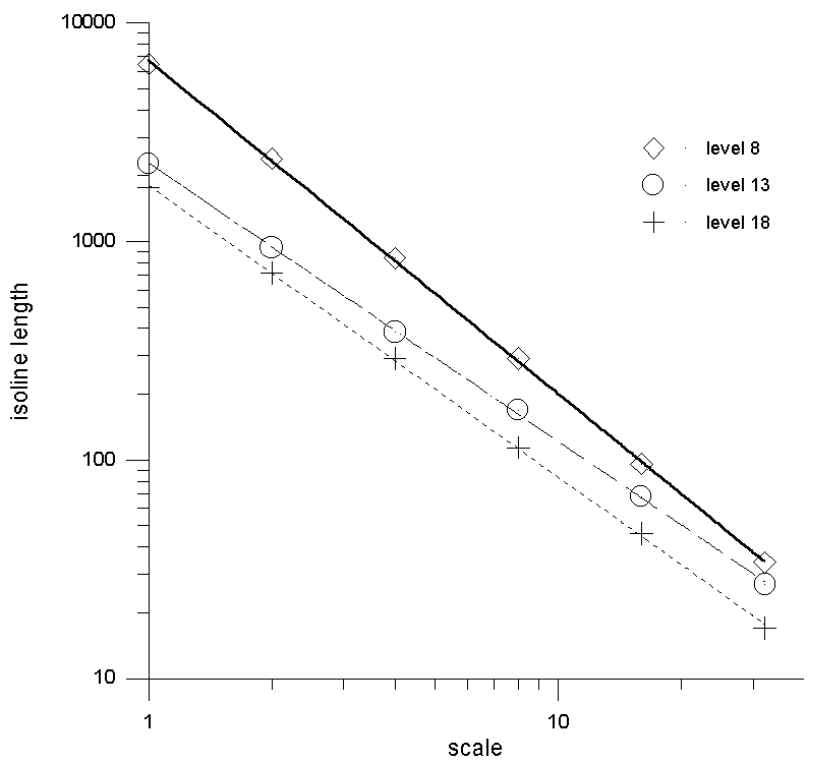

Fig. 3. Number of boxes covering the isoline as a function of box scale for isolines presented in Fig. 2.

Using substitutions from Eq. (2) and Eq. (3) to Eq. (5):

$$
\begin{aligned}
& N_{1}(\delta)\left(1-D_{1} \frac{d \delta}{\delta}\right)+N_{2}(\delta)\left(1-D_{2} \frac{d \delta}{\delta}\right) \approx \\
& \left(N_{1}(\delta)+N_{2}(\delta)\right)\left(1-D_{1+2} \frac{d \delta}{\delta}\right) .
\end{aligned}
$$

Now one can simplify the expression and obtain:

$$
N_{1}(\delta) D_{1}+N_{2}(\delta) D_{2} \approx\left(N_{1}(\delta)+N_{2}(\delta)\right) D_{1+2},
$$

from which Eq. (1) follows directly.

One can see that in the limit of $\delta \rightarrow 0$ we would have $D_{1+2}(\delta) \rightarrow \max \left(D_{1}, D_{2}\right)$. The proposition is obviously generalized on a case of the greater number of auroral forms. Therefore, for finite scale $\delta>0$ we can be sure that the great structure near zenith of an all-sky image will be the main contributor in the integral dimension value. Other structures near the horizon will have a relatively smaller weight.

Generally, it is not obvious that the dependence $\log N(\delta, I)$ on $\log \delta$ is close to the linear one. Figure 3 shows some examples of such dependences for isolines discussed above. One can see that these dependences, especially for small $\delta$, are practically linear. The linear approximating was conducted by the least-squares method, and the discrepancy of the dimension value does not exceed 0.05 . If it is not stipulated, this estimation will be implied in all further results.

\section{Testing by model images}

Now we discuss the numerical testing of the box-counting dimension algorithm using the model images with the known fractal characteristics. The necessity of testing is urged by several reasons: first, the range of possible $\delta$ scales is not 


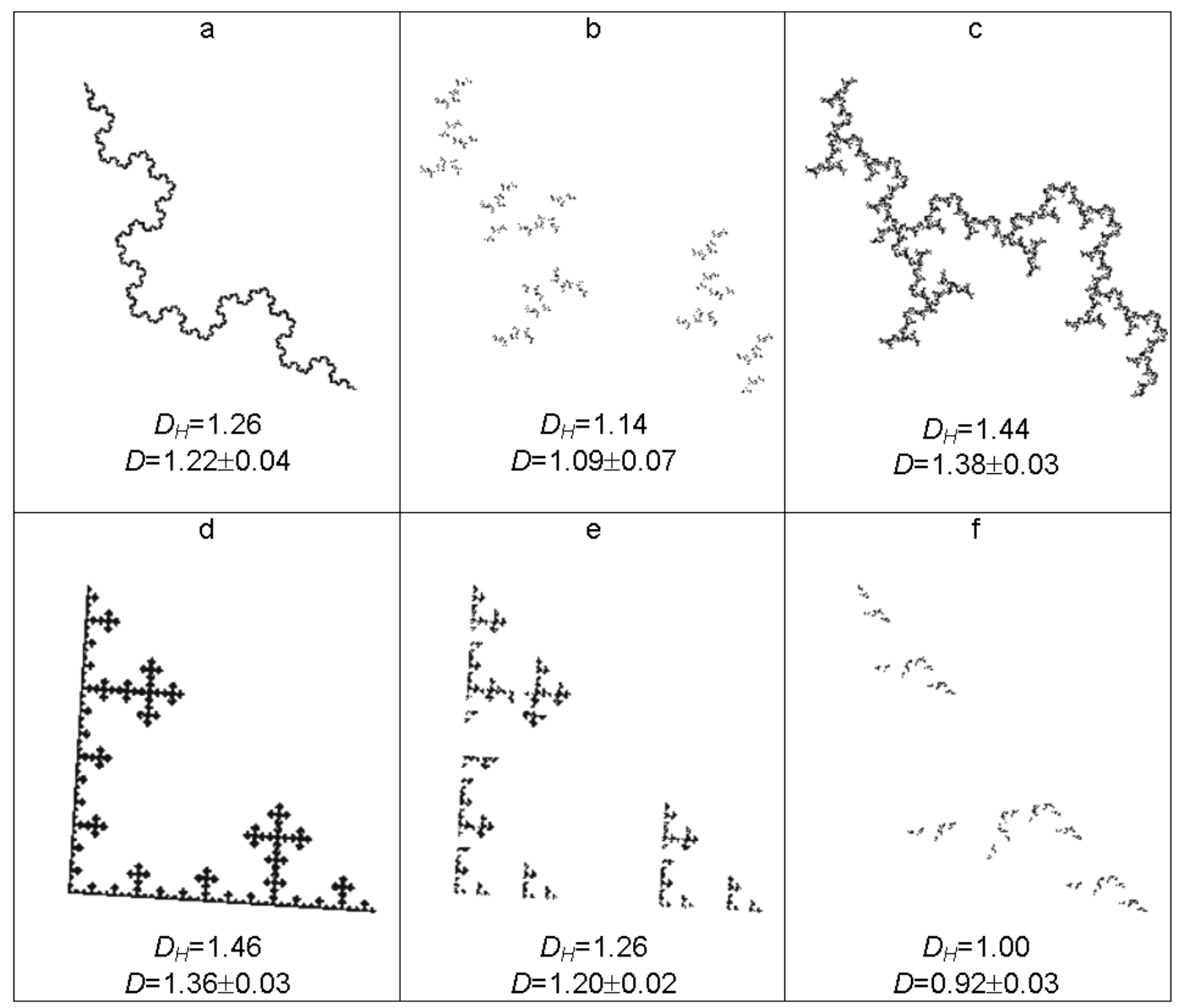

Fig. 4. Examples of the regular prefractal structures: $D_{H}$ - theoretical fractal dimension; $D$ - box-counting dimension calculated by the image.

great; second, the projection of the auroral emission area in an all-sky image strongly distorts the spatial scales; and, third, the correspondence between the fractal dimension of the set and the dimension of its boundary is not always obvious (Falconer, 1995). The testing of the algorithm was conducted over many steps and we will shortly illustrate each step.

\subsection{Regular prefractals}

For this test the images of different regular prefractals were constructed on a grid $256 \times 256$ by the obvious from a figure recursive procedure with 5 iterations. At such spatial resolution the prefractal image practically does not differ from the image of the appropriate regular fractal. The capability of an estimation of fractal dimension value by the box-counting algorithm was tested. The examples of the obtained results are shown in Fig. 4. In the figure the theoretical dimensions of fractals $\left(D_{H}\right)$ and box-counting dimensions calculated by the model image are presented. One can see that the algorithm confidently evaluates expected fractal dimension by the model image. There is a tendency towards underestimation of the dimension, on the average, by 0.04-0.06 compared to the theoretical dimension.

\section{2 "All-sky" projection of prefractals}

An important property of fractals is that the fractal dimension is an invariant for the wide class of transformations, namely the so-called bi-Lipschitz transformations (see details in Falconer, 1995, Corollary 2.4b). Basically, the mapping determining a projection of auroral glow in a TV frame by fish-eye ("all-sky") camera, obeys these conditions at all points, except the points appropriate to directions which are perpendicular to geomagnetic field lines. However, for prefractals on a grid with the finite resolution the invariance of box-counting dimension is not obvious. The results of numerical testing are shown in Fig. 5 for the images of the regular prefractals, transformed by "all-sky" projection. When constructing the images it was presumed that the structure altitude is $100 \mathrm{~km}$ above the Earth's surface. One can see that the estimation of the dimension by the box-counting algorithm practically does not differ from the dimension for the distortionless images. However underestimation of the dimension value is larger than in the previous test, and it can reach $\sim 0.1$ for dimensions $>1.4$. 


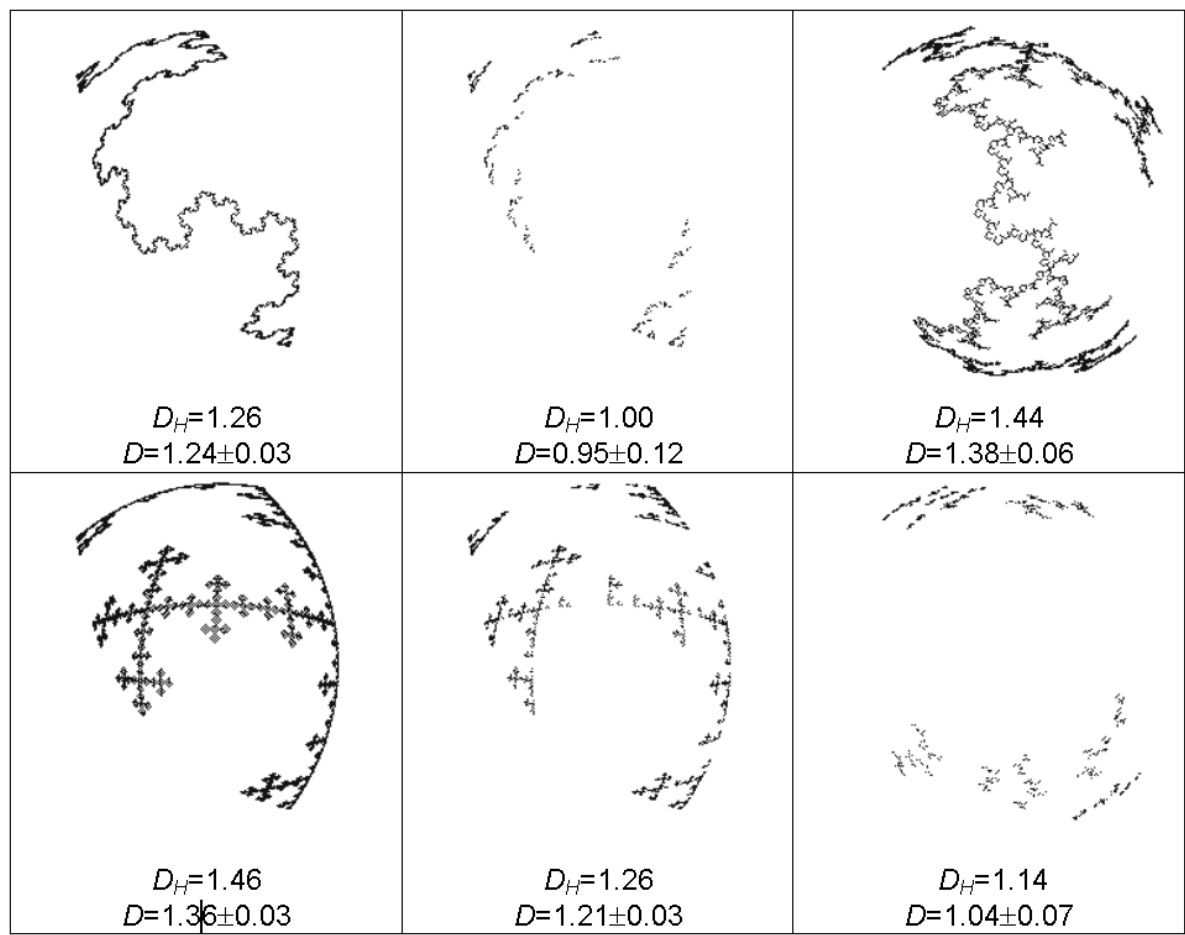

Fig. 5. Examples of the regular prefractal structures, transformed by all-sky projection: $D_{H}$ - theoretical fractal dimension; $D$ - box-counting dimension calculated by the image.

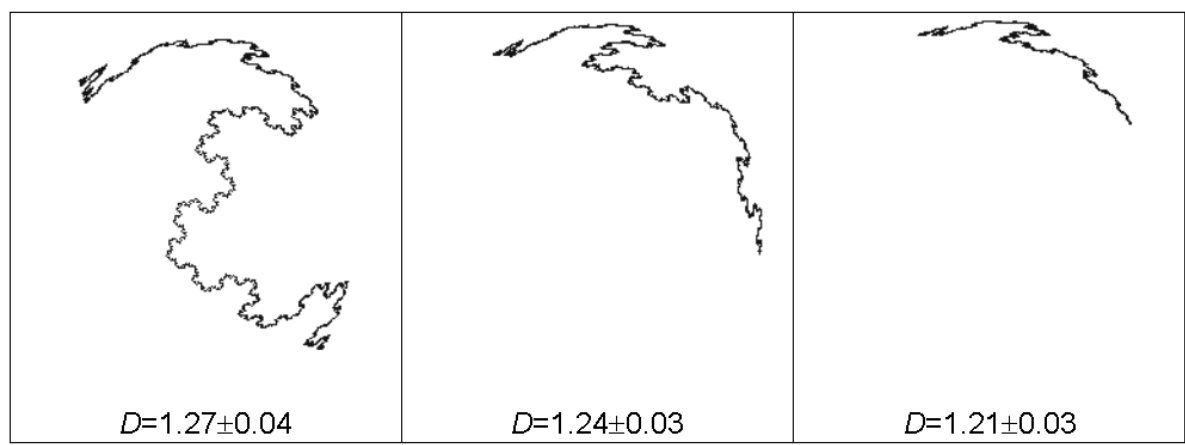

Fig. 6. Testing of dependence of the box-counting dimension on structure position.

\subsection{Position of the structure in the sky}

It seems obvious that the details in the image obtained by the all-sky camera become less discernible with the motion of the auroral structure to the horizon; therefore, the results of the dimension calculations depend on the position of the auroral structure in the image. The results of testing of this reason are shown in Fig. 6. One can see that the calculated box-counting dimension slowly depends on the position of the structure in frame. The expected tendency towards decreasing of the dimension to 1.0 (i.e. loss of details of structure) at the structure movement to horizon has an effect only directly near the horizon.

\subsection{Noised image with altitude distribution of emission}

To simulate the more realistic images we used the uniform auroral arcs with the preset fractal structure and altitude emission distribution which is typical for arcs in the polar aurora. However, the television signal contains different distortions associated with features of the recording system and activity of different self-tunings at the record. Here we shall simply consider, that the distortions consist of two components: (1) additive random noise with normal distribution and (2) distortions circumscribed by a hardware function of the recording system. It is possible to consider that this function is rather smooth in frame in an effective range of intensities and has little time variation. Therefore, this distortion has no influence on the dimension calculation, and only the random 

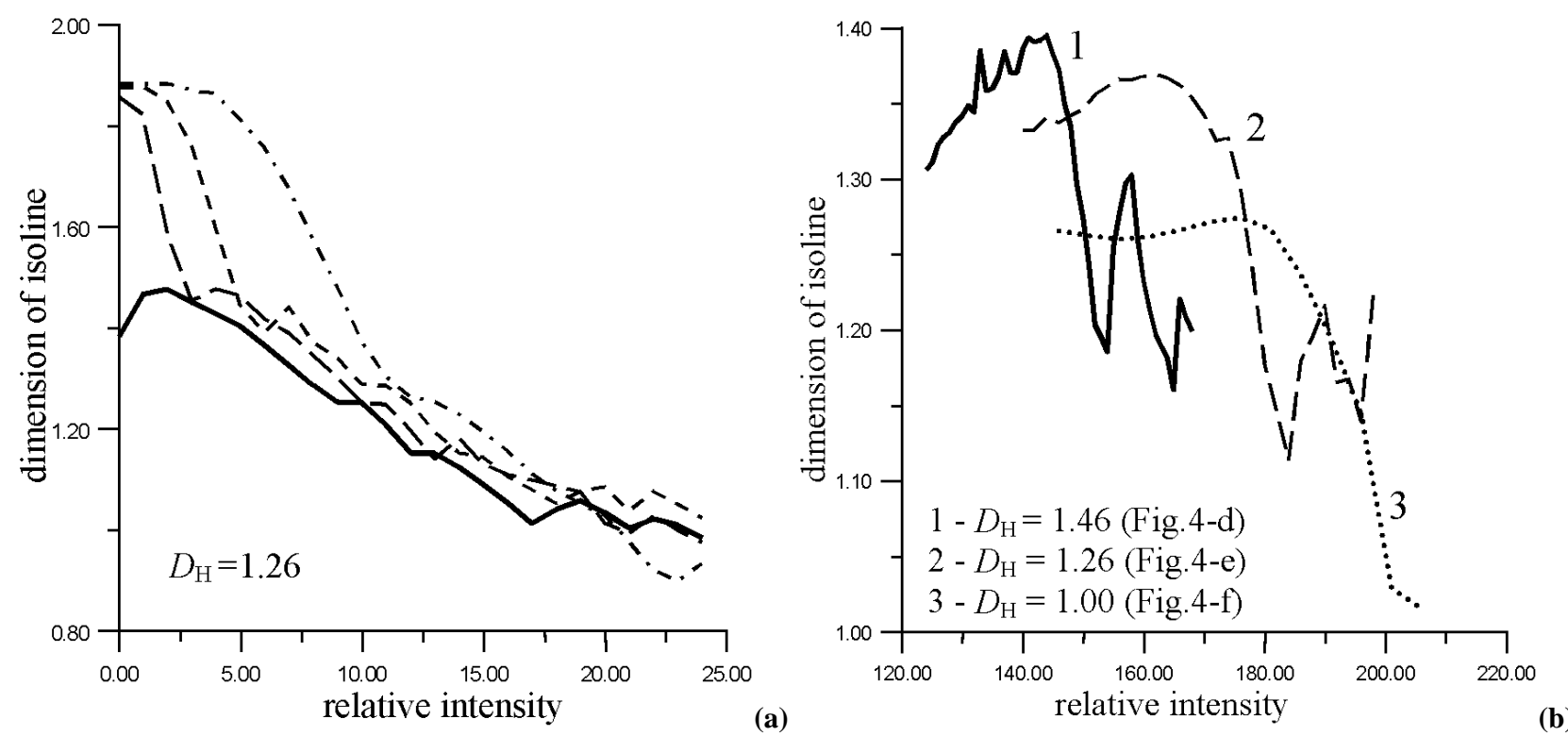

Fig. 7. Dependence of the isoline dimension spectrum on the noise: (a) linear sensitivity scale of model image, solid line - without noise, other lines - noise with zero mean and dispersions of 1, 4 and 9 units of intensity, respectively; (b) logarithmic sensitivity scale. $D_{H}-$ theoretical fractal dimension. References to Fig. 4 show the fractal structure used for image modelling.
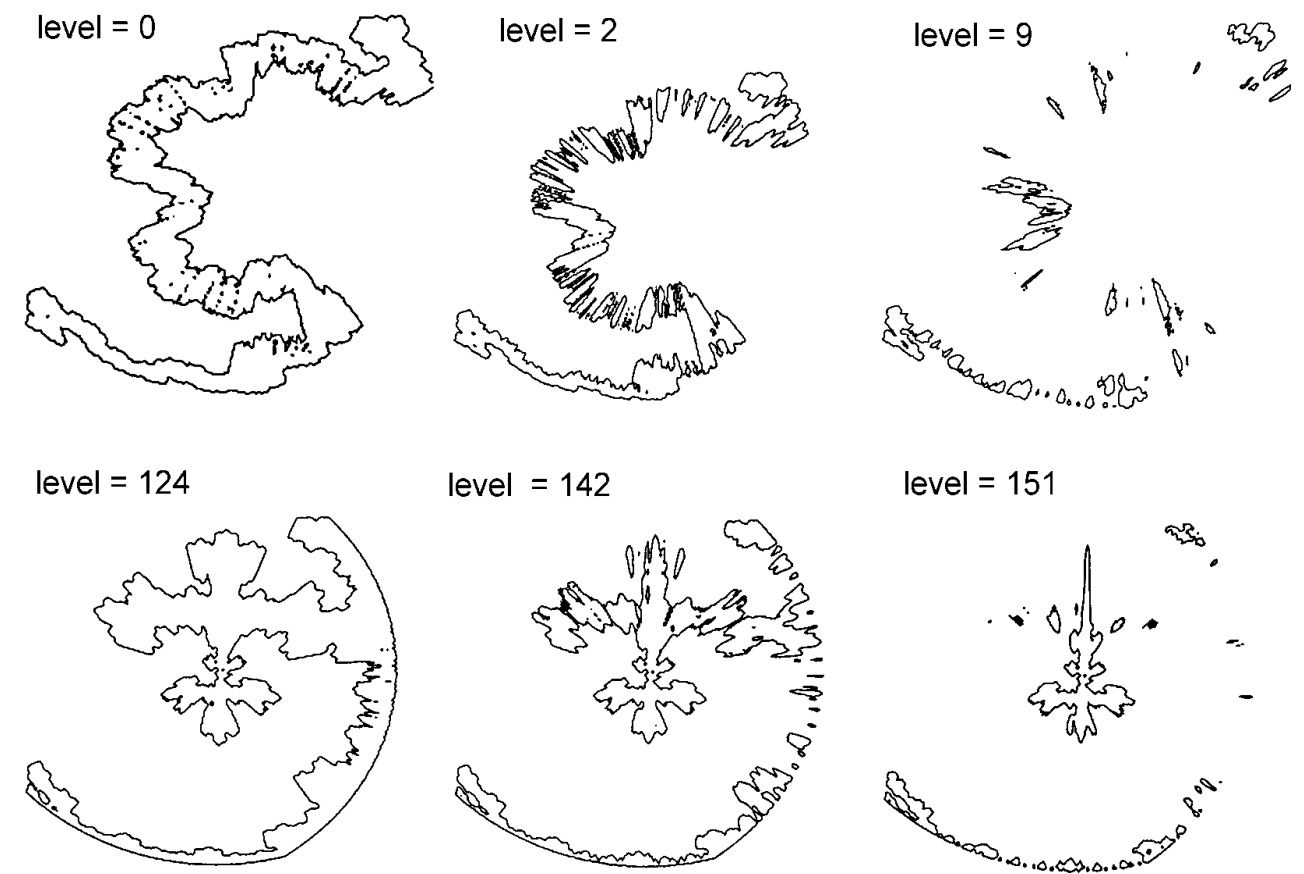

level $=151$
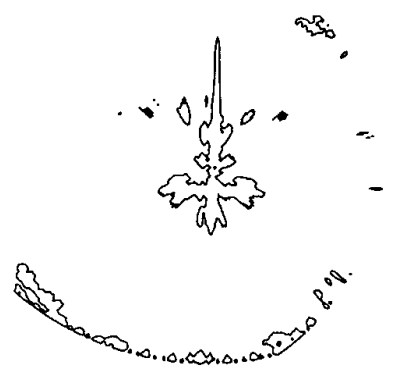

Fig. 8. Examples of isolines used in box-counting calculations of the spectra presented in Fig. 7: (a) solid line from Fig. 7a; (b) solid line from Fig. 7b.

noise in small intensities is assumed.

By the model images the capability of finding "the most complex isolines" (MCI) was tested. The MCI region is expressed in a maximum on the curve $D(I)$. Some examples of the obtained spectra of isoline dimension are shown in Fig. 7. The arc structure used is presented by reference to
Fig. 4 and dimension of associated regular fractals. The isolines for some characteristic levels of intensity are adduced in Fig. 8. The existing television cameras at the expense of activity of self-turning systems have different sensitivity depending on the light intensity. Usually, the cameras have a linear scale of sensitivity, for some of them the sensitivity 

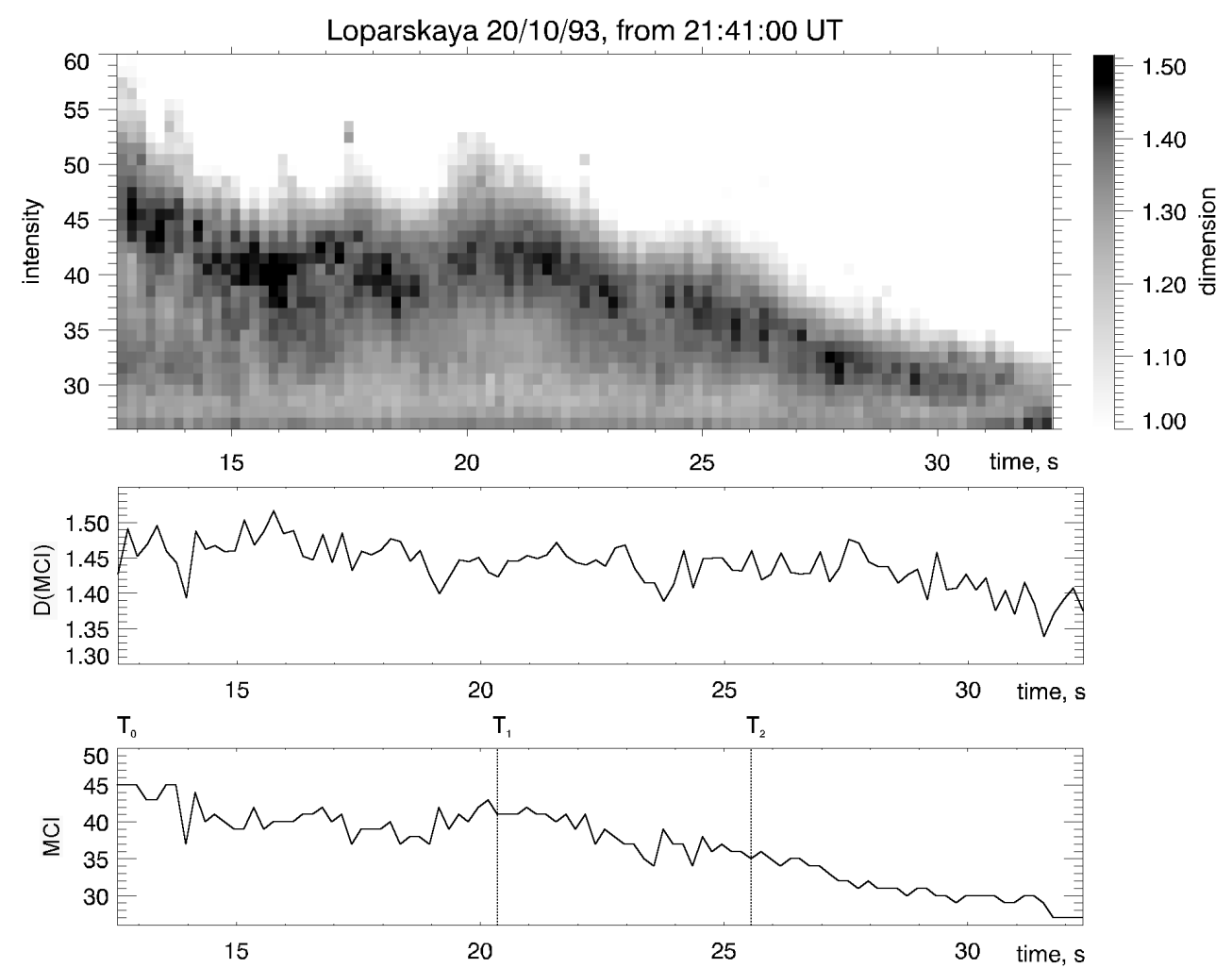

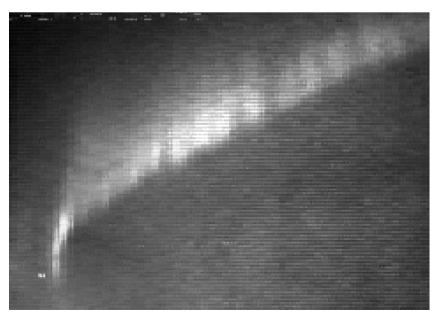

$\mathrm{T}_{0}$

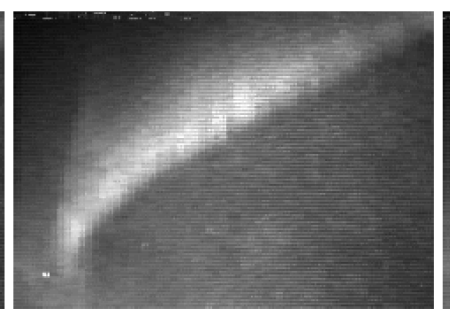

$T_{1}$

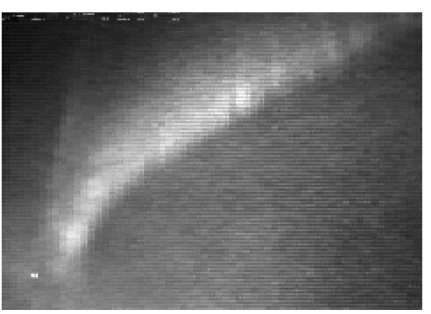

$\mathrm{T}_{2}$

Fig. 9. Analysis of the auroral dynamics during event 1. Panels from top to bottom: (a) temporal evolution of the isoline dimension spectrum; (b) dependence of the greatest isoline dimension with time; (c) dependence of the intensity corresponds to "the most complex isoline" (MCI) with time; (d) images associated with the moments, marked in the previous panel.

scale is nonlinear, close to logarithmic. The nonlinear scale allows one to register the greater range of intensities without saturation of the TV tube, and in this sense it is preferable for aurora observation. We consider both situations. Figure $7 \mathrm{a}$ shows IDSs calculated for model images with linear sensitivity scale. The noise is assumed to have the normal distribution with zero mean and dispersions of 1, 4 and 9 units of intensity, respectively. One can see that significant noise can completely disguise the maximum on the curve of $D(I)$. For comparison Fig. 7b presents IDSs obtained for model images with logarithmic sensitivity scale. One can deduce that for the images with a nonlinear (logarithmic) scale of intensities the maximum on the $D(I)$ curve is better seen. In addition, though the dimension value in the maximum does not coincide with the dimension of the regular fractal used for image simulation, nevertheless the larger value at the maximum of the $D(I)$ curve corresponds to the larger dimension of the fractal.

\section{Analysis of auroral dynamics}

In previous sections the definition and the testing of the fractal approach for describing of the auroral structure have been discussed. The approach is based on the calculation of the spectrum of the box-counting dimension of isolines in the auroral image. Now we consider the possibilities which the approach gives us for the analysis of auroral dynamics. We shall try to follow the auroral dynamics by the evolution of the isoline dimension spectrum during several typical auroral events.

For the analysis we use the data of ground-based TV observations, which was provided by the Loparskaya station during 1993-1994 by a narrow angle lens camera (angle of 

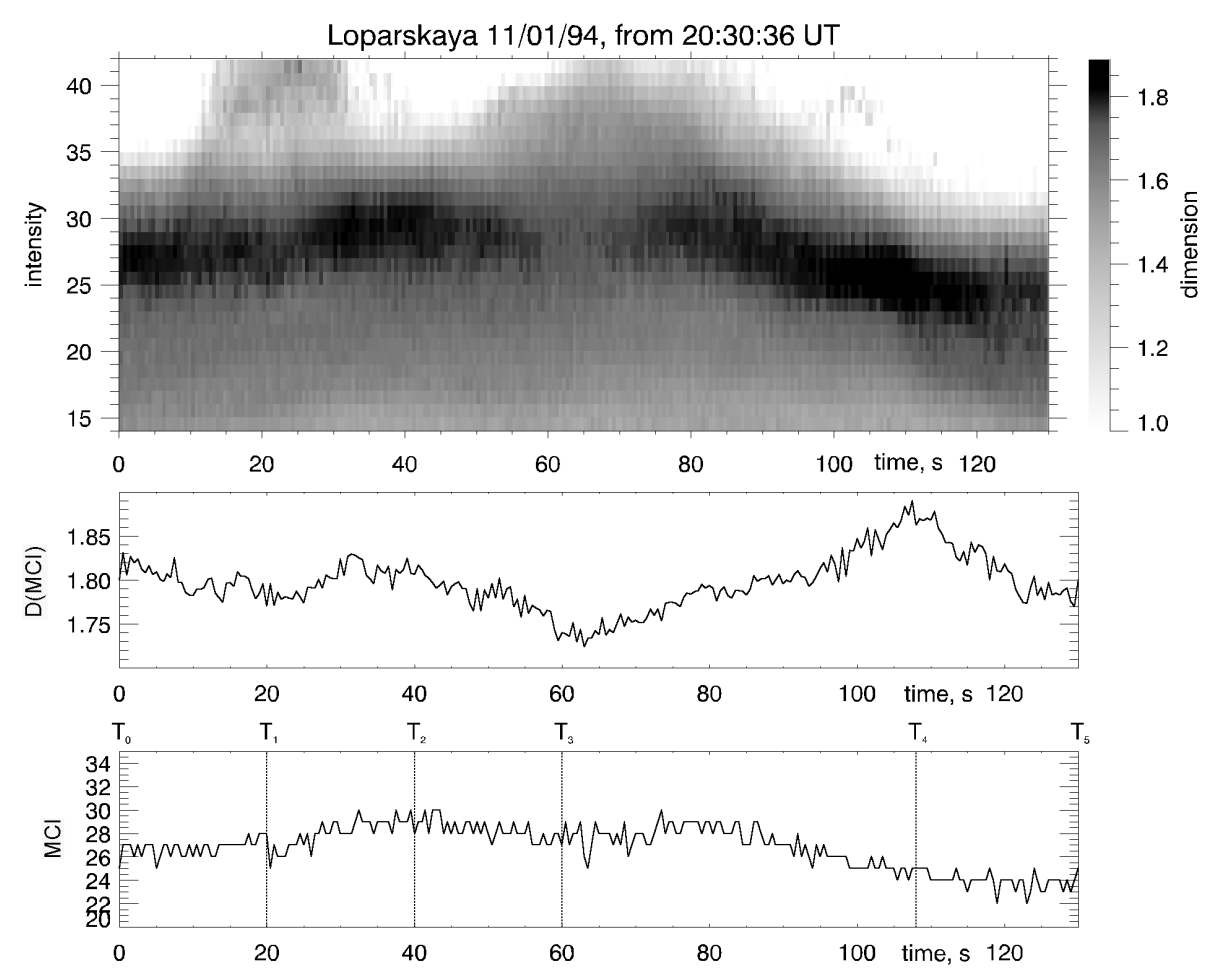

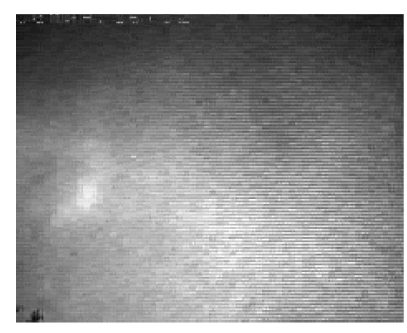

$\mathrm{T}_{0}$

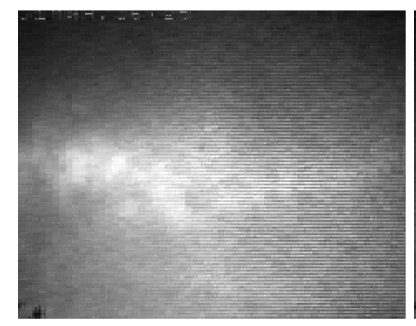

$\mathrm{T}_{3}$

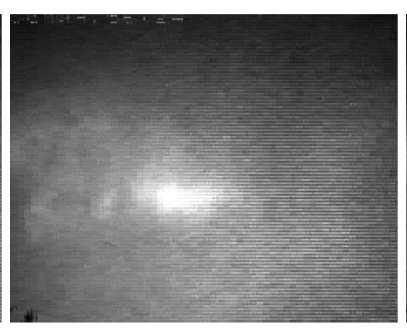

$\mathrm{T}_{1}$

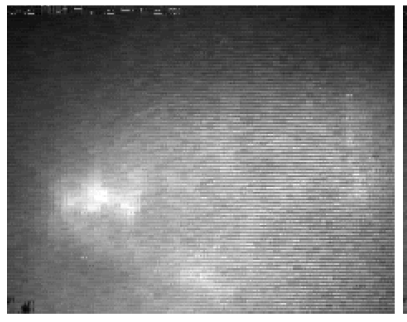

$\mathrm{T}_{4}$

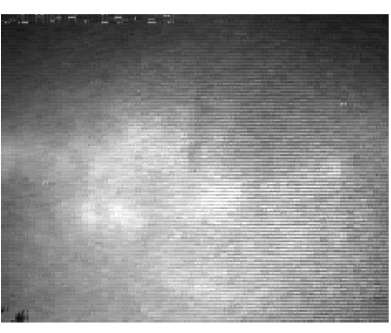

$\mathrm{T}_{2}$

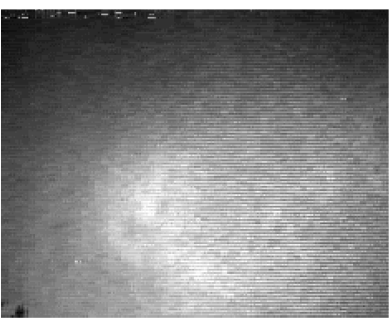

$\mathrm{T}_{5}$

Fig. 10. The same as Fig. 9, but for event 2 .

view was $\sim 30^{\circ}$ ). The application of narrow angle lens allows one to increase the spatial resolution of the fine auroral structure to $<0.5 \mathrm{~km}$. Unfortunately, the utilization of the greater part of the records was complicated by the presence of a regular external modulation and saturation in intensive lights. Five intervals which do not have these distortions have been selected for the analysis. The TV data have been digitized by a standard PC frame-grabber with spatial resolution of $255 \times 255$ points, time resolution of 5 frame/s and 64 intensity levels.
The results of processing these sets of frames are shown in Figs. 9-14. Each figure contains (from top to bottom):

(a) The $D(I, t)$ panel - temporal evolution of the $D(I)$ spectrum, the value of isoline dimension is shown by grey gradations in axes of time - intensity.

(b) Dependence of the greatest isoline dimension with time. This dimension is associated with the intensity for "the most complex isoline", therefore, we refer to it as $D$ (MCI). A similar dependence for a local maximum 

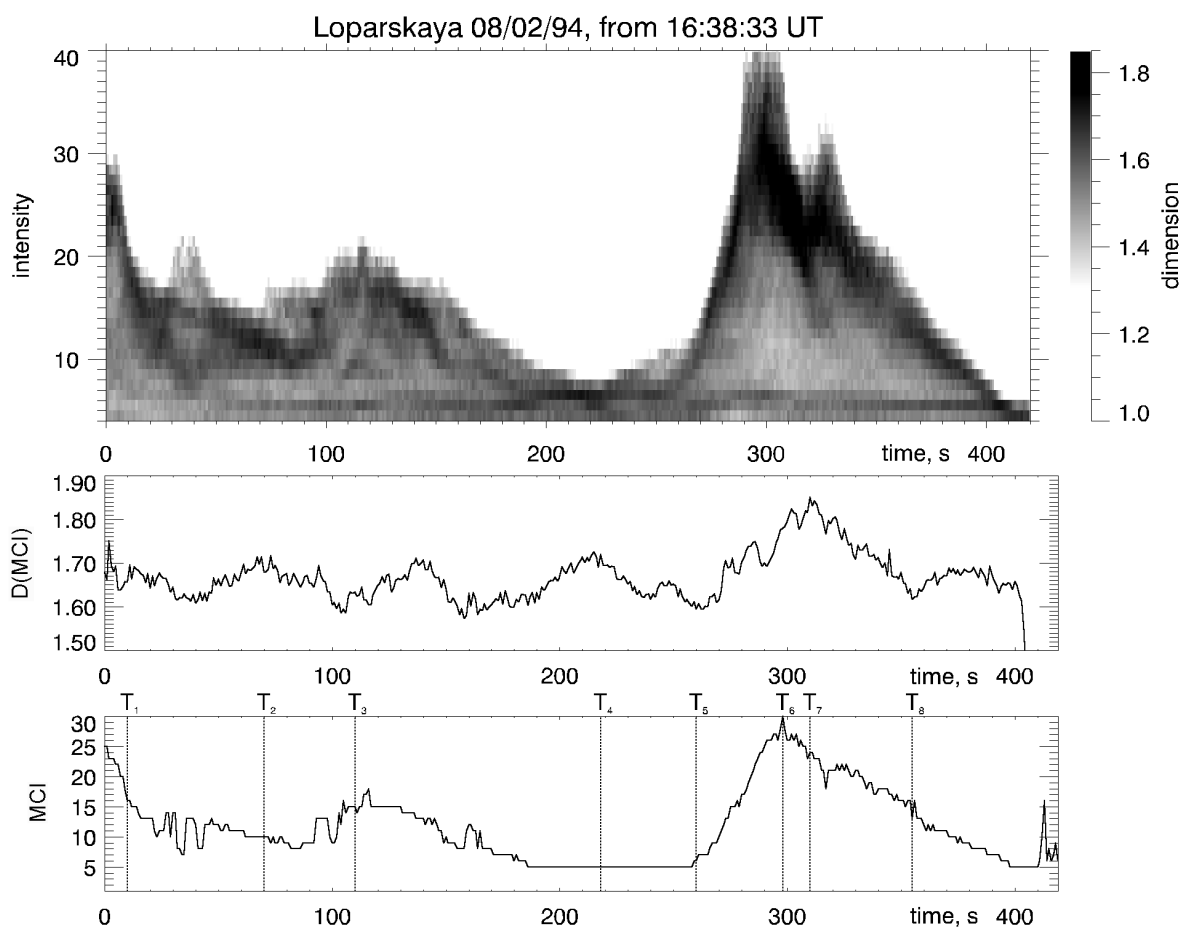

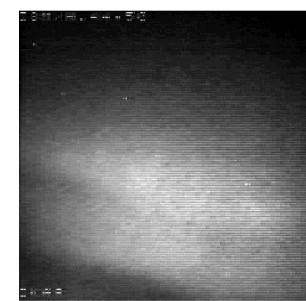

$\mathbf{T}_{1}$

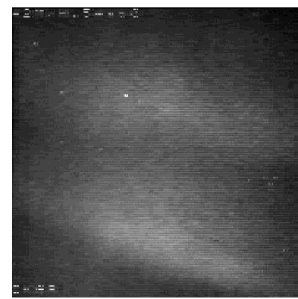

$\mathbf{T}_{5}$

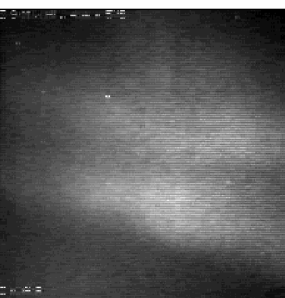

$\mathrm{T}_{2}$

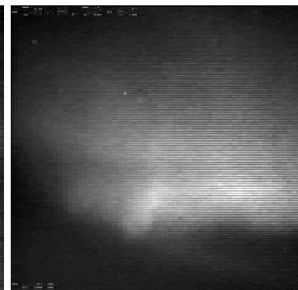

$\mathrm{T}_{6}$

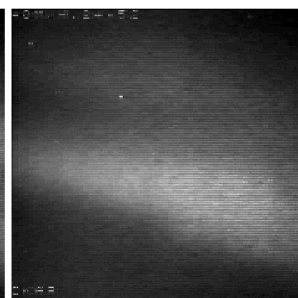

$\mathrm{T}_{3}$

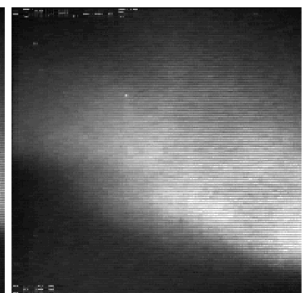

$\mathbf{T}_{7}$

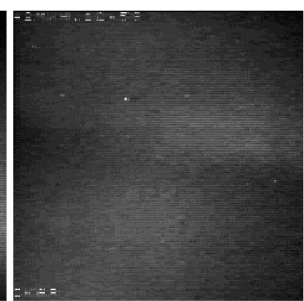

$\mathrm{T}_{4}$

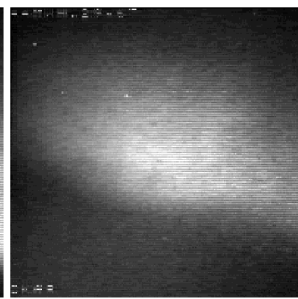

$\mathrm{T}_{8}$

Fig. 11. The same as Fig. 9, but for event 3 .

at large intensities is marked in Fig. 12 and Fig. 13 by crosses.

(c) Dependence of the intensity for MCI with time, we refer to it as $I(\mathrm{MCI})$. The position of intensity appropriate to a local maximum is shown by crosses. Some moments in this panel are marked by dotted lines.

(d) TV images are displayed which are associated to the moments, marked in the previous panel.

Let us consider the events in detail, in ascending order of complexity and we shall try to observe as the changes in the auroral glow appear in the dynamics of local maxima in the isoline dimension spectrum and intensities, associated with maxima.

Event 1, Fig. 9

The images contain a stable arc with ray structure. According to Hallinan and Davis (1970), the rays in active auroral arcs are curls. In the beginning of an event the arc is bright, the structure is well observed, the maximum value of the dimension is $D=1.45-1.5$ at intensities $I=40-45$. Gradually, the arc dies away and rays spread. This evolution is expressed by the decrease in the maximum dimension to $D=1.35-1.4$ and intensity to $I=30$.

Event 2, Fig. 10

Diffuse spots. During the considered 130 s, the spots greatly 

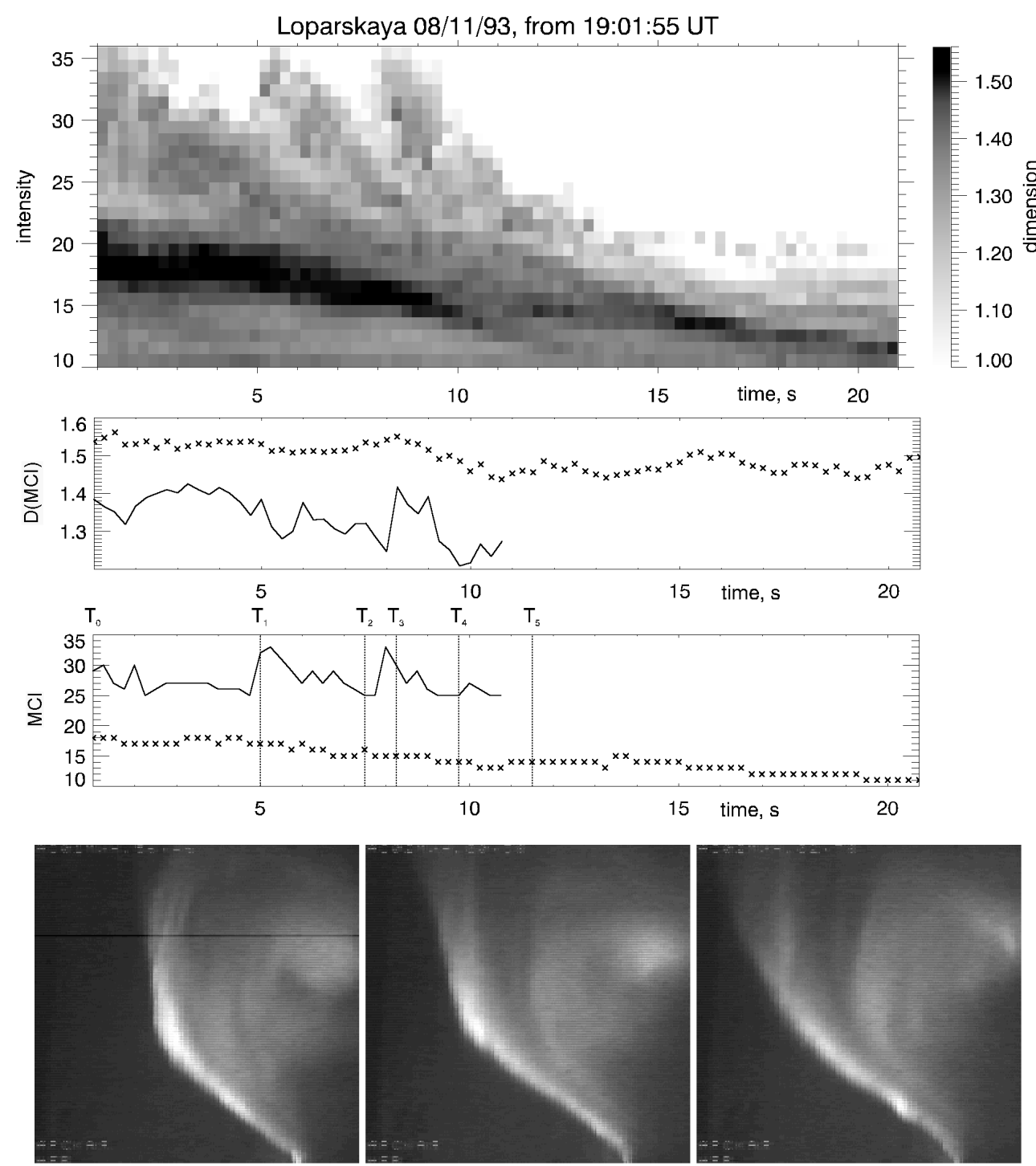

$\mathrm{T}_{0}$

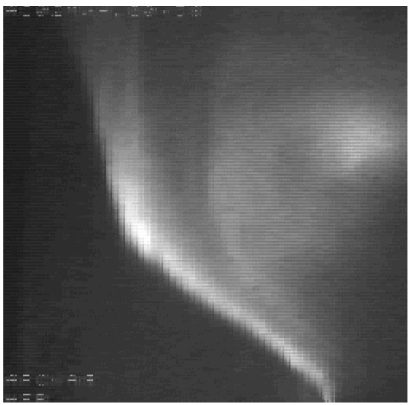

$\mathrm{T}_{1}$

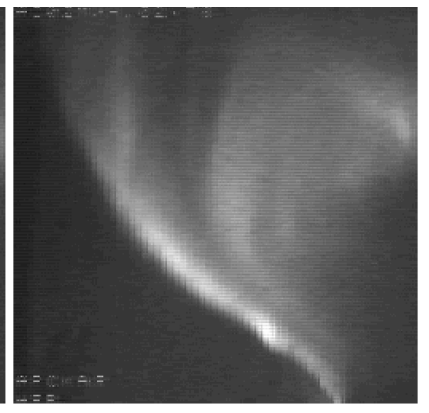

$\mathrm{T}_{2}$
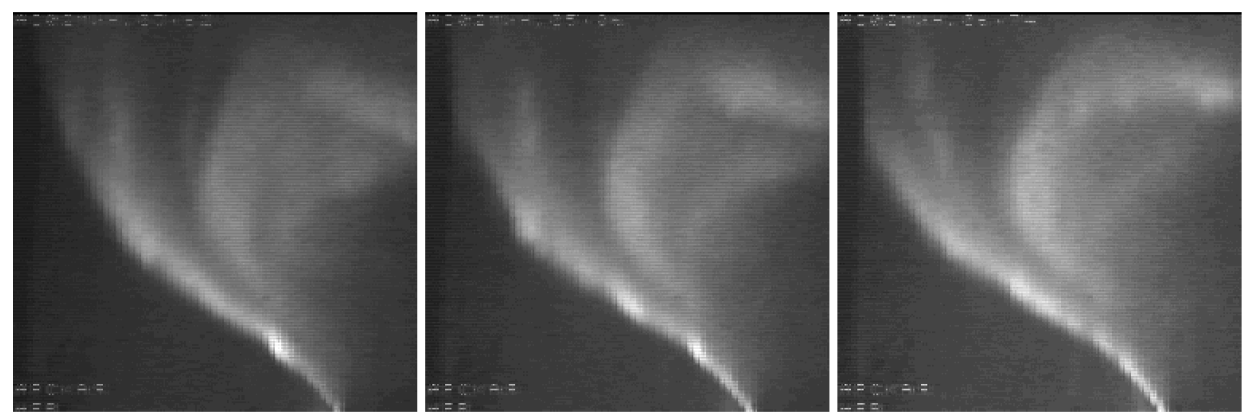

$T_{3}$

$\mathrm{T}_{4}$

$\mathrm{T}_{5}$

Fig. 12. The same as Fig. 9, but for event 4. Dependences for the local maximum at large intensities and for the intensity associated with the local maximum are marked in panels (b) and (c) by crosses.

change their form and position. Nevertheless, $I(\mathrm{MCI})$ varies very smoothly and slowly. At the same time, the maximum dimension varies within rather broad limits: from 1.73 to 1.88 , that is the changes happen just in the structure of the auroral glow, rather than in intensity. The appearance of brighter localized spots (15-30 s and 50-80 s) is seen in dependence $D(I, t)$ at $I>35$ and is accompanied by a decrease in the maximum dimension at $I<35$. Let us mark 
essentially large values of the maximum dimension in this case compared to the first event.

Event 3, Fig. 11

Diffuse glow. In the beginning of the interval the auroral glow looks like two diffuse bands without any fine structure. During the first $250 \mathrm{~s}$, the emission intensity was changing significantly and practically merged with the background by the time $I=200 \mathrm{~s}$. The maximum dimension smoothly oscillated (with a period of about $70 \mathrm{~s}$ ) within the limits of 1.6-1.7. After the moment $T_{5}(260 \mathrm{~s})$ a strong brightening of the aurora happened, and for some time the small-sized brighter spots occurred, that led to the increase in the maximum dimension to 1.85 . After the moment $T_{8}$ the glow returns to its initial state and gradually dies away. It is possible to note, that during this event, compared to the first one, there are essential variations as maximum dimension $D(\mathrm{MCI})$, and $I(\mathrm{MCI})$, and these variations are not correlated.

Event 4, Fig. 12

During the first $5 \mathrm{~s}$ of this event, the bright arc is moving from the middle to the left side of the image. Behind the arc there is a diffuse band. Later, the two waves of brightening are running along the arc (in the image from top to bottom), after that the arc intensity is decreasing. The waves of brightening have been identified according to Hallinan and Davis (1970) as folds. On the $D(I, t)$ panel it is possible to note the regions having different dynamics: (1) At $I<20$ there are smooth time variations of the maximum dimension $(D=1.45-1.55)$ and $I(\mathrm{MCI})$; this area characterizes mainly the diffuse band behind the arc. (2) At $I>20$ on the $D(I, t)$ panel there is a more dynamical local maximum of dimension $(D=1.25-1.4)$. This maximum follows the movement of bright structures. For the motion along the arc the waves of brightening run to the horizon and decrease in the size, where $D(I, t)$ displays such motion as a decrease in $I(\mathrm{MCI})$. Event 5, Fig. 13

This event is a typical evolution of the auroral arc in the background of diffuse glow. As in the previous case on the $D(I, t)$ panel it is possible to select two regions having different dynamics. At $I<25$ there is a smooth growth of the maximum dimension from $D=1.7$ up to $1.8, I(\mathrm{MCI})$ is also increasing a little; this area characterizes the diffuse glow. The structure evolution of the arc is mirrored by the $D(I, t)$ at $I>25$. The moment of $T_{0}$-the arc is bright without noticeable fine structure, $D(\mathrm{MCI})=1.55$. The moment $T_{1}$-region of bright emission on the arc is divided into two parts and becomes more precise, and the maximum dimension decreases to 1.38 . The moment $T_{3}$-a gap starts to occur on the arc, and the maximum dimension is magnified up to 1.55 . Then the arc damps and breaks up into rays $\left(T_{4}, T_{5}\right)$. The number of rays gradually decreases $\left(T_{6}, T_{7}\right)$, and the arc merges with background glow $\left(T_{8}\right)$.

The ranges of dimension values for the most complex isoline, $D(\mathrm{MCI})$, obtained for discussed types of auroral structures, are given in the Table 1 . Since all data were recorded by the same instrumentation, there is a possibility to compare the obtained dimensions for the considered events. One can see that the dimensions have the expected tendency: the dif- fuse structures are more complex, and therefore, they have the dimension value above, rather than the discrete one. One exception has occurred for the diffuse band in the fourth event. The detailed additional consideration has shown, that in this case, isolines for $I<22$ consist of a strongly structured part from a diffuse glow and a large, smooth linear part along the border of the bright arc. The dimension of the full isoline is a weighed-mean value (see Proposition in Sect. 3), therefore, it is a little bit lower than in the other cases. The range of dimension values calculated without the linear part of the isolines is given in the table in brackets. From the considered examples of $\mathrm{TV}$ data processing it is possible to make the following conclusions:

1. For all considered series of TV frames the isoline dimension spectra for adjacent frames vary smoothly, and this allows one to use dependences $D(I, t)$ for the description of the auroral dynamics.

2. The maxima in the isoline dimension spectra is associated with the structures in the image, thus the change in maxima value and intensities associated with them reflects the dynamics of the auroral structure.

3. The obtained dimensions for different auroral events have the expected tendency: the diffuse forms have thedimension value above, rather than discrete structures.

\section{Conclusions}

The paper provides a definition and testing of the fractal approach for the description of the auroral structure. The approach is based on the calculation of the spectrum of the boxcounting dimension of isolines in an auroral image. Then an auroral form is usually present in the image; there are three important regions in the spectrum $D(I)$ curve: (1) the region of noised background intensities; (2) the region with the most complex isolines (MCI); and (3) the region of separation between them. The MCI region corresponds to the most complex structure in the auroral form, and the dimension value for the $\mathrm{MCI}, D(\mathrm{MCI})$, is usually greater than 1.25 . For the region of separation the dimension value is smaller and usually in this region there is an intensity with minimal dimension. The isoline for this intensity separates the auroral form from the noised background. In the region of the noised background the dimension value increases with the intensity decrease.

From the above presented results we can summarize that the description of the auroral image by the spectrum of isolines dimension allows us:

- to localize an auroral structure in the background glow;

- to characterize numerically the spatial structure of the aurora;

- to select the range of intensities associated with the most developed auroral structures; 


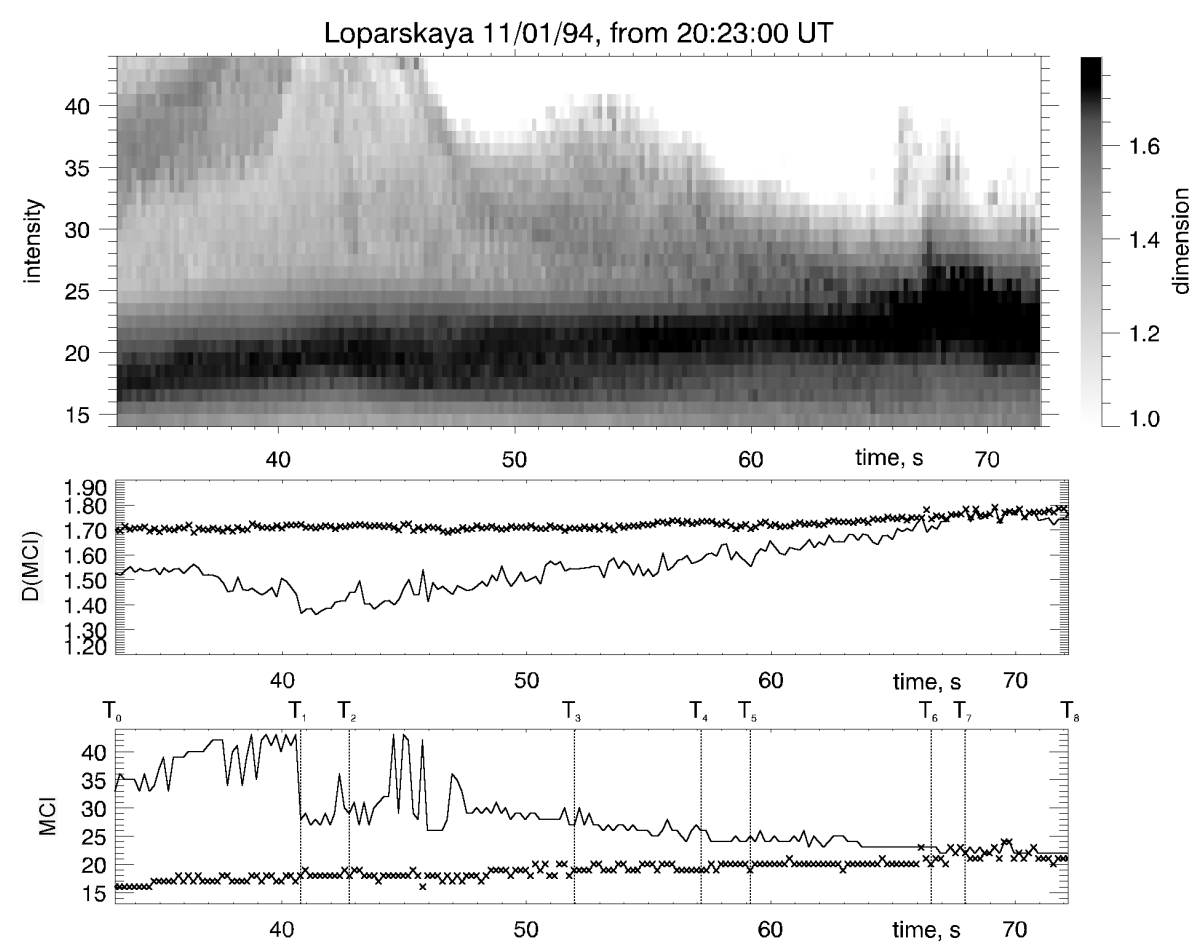

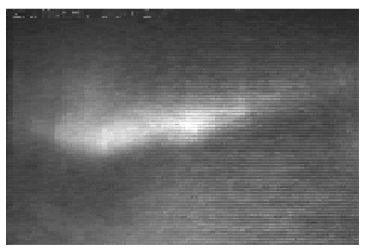

$\mathrm{T}_{0}$

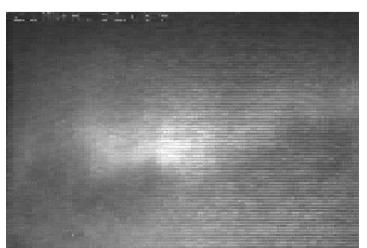

$\mathrm{T}_{3}$

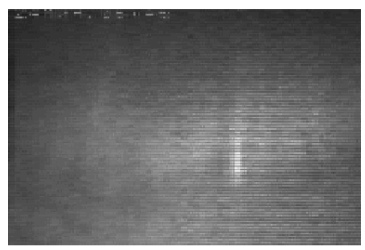

$\mathrm{T}_{6}$

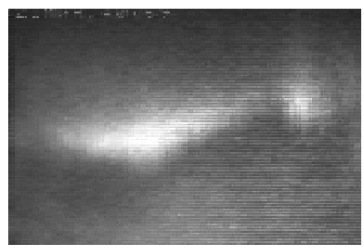

$T_{1}$

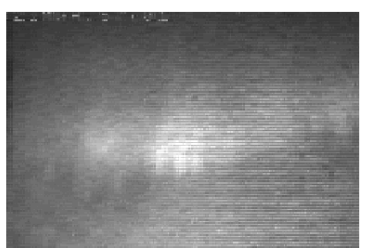

$\mathrm{T}_{4}$

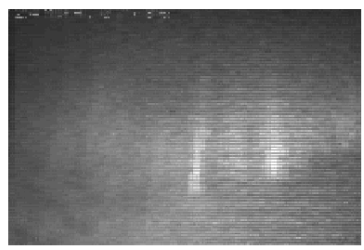

$\mathrm{T}_{7}$

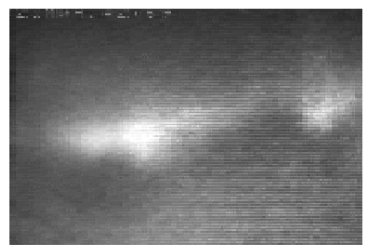

$\mathrm{T}_{2}$

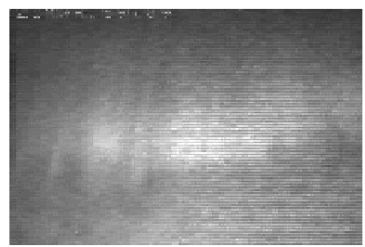

$\mathrm{T}_{5}$

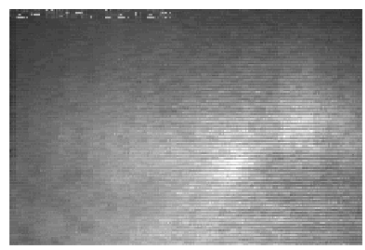

$\mathrm{T}_{8}$

Fig. 13. The same as Fig. 12, but for event 5 .

- to observe the dynamics of the auroral structure development.

This description can give us an essential supplement to the standard methods of auroral data processing, as the obtained characteristics of the image (IDS, $I(\mathrm{MCI})$ and $D(\mathrm{MCI})$ ) enable us to more precisely use the information about the spa- tial distribution of the auroral glow, rather than standard methods.

In an application of the suggested technique to the auroral physics we see two possible approaches. First, the dimension of the most complex isoline, $D(\mathrm{MCI})$, can be used as a structure index of the aurora. This index can be used to character- 
Table 1. Dimension of the most complex isoline for different auroral events

\begin{tabular}{lccccc}
\hline Event number & 1 & 2 & 3 & 4 & 5 \\
\hline Diffuse bands & - & - & $1.6-1.85$ & $\begin{array}{r}1.45-1.55 \\
(1.55-1.72)\end{array}$ & $1.7-1.8$ \\
\hline Diffuse spots & - & $1.73-1.88$ & - & - & - \\
\hline Rayed arc & $1.4-1.5$ & - & - & - & $1.55-1.65$ \\
\hline Arc without rays & - & - & - & $1.25-1.4$ & $1.35-1.5$ \\
\hline
\end{tabular}

ize both separate structure, and the entire neighbourhood of a view point (by all-sky image).

Second, in some cases it is possible to associate the changes in the $D(I)$ spectrum with some physical processes happening in the magnetosphere-ionosphere system. An aurora is the manifestation of these processes, and it is assumed that the auroral structure is connected with the structure of the spatial region of these processes action. The dimension may be used as a numerical characteristic of spatial irregularity of the region. The dimension of $\mathrm{MCI}$ on the $D(I)$ curve would be connected with the main region of this irregularity. When we see an increase in this dimension, this may be a reason to talk about the development of the irregularity. Therefore, we can suppose that the variation of the MCI dimension expresses the dynamics of the nonlinear dissipative system which describes this irregularity developing. However, for the detailed study it is necessary to carry out a careful selection of events, and also the engaging of the data of other measurements and an appropriate physical model. The most obvious phenomena requiring such an approach are: (1) polar boundary of the discrete aurora, especially during the transition from the growth phase to the explosive phase of substorm; (2) pulsing spots and patches in the morning sector and their connection with VLF waves; (3) ray structures of aurora. Some steps in this direction were made in our work (Kozelov et al., 2002).

Also, we can note several numerical applications of fractal features to TV image processing. First, using the $D(I)$ curve we can select better the intensity limits of digitizing. Second, the results of the dimension calculation may be used for the mapping of all-sky images to geographic map. The generally used mapping algorithms produce non-realistic images, because the altitude distribution of the aurora emission is mapped as ray-like spatial structures on the plane. The MCI may be used as a limit to decrease this problem. Third, the dimensions that correspond to MCI may give a theoretical limit of the auroral image compression without the loss of important information.

Acknowledgements. The author is thankful to V. R.Tagirov (Polar
Geophysical Institute, Apatity, Russia) for the data of TV observations by a narrow angle lens camera. The work was partly supported by the Russian Foundation for Basic Researches, grant 0105-64827.

Topical Editor T. Pulkkinen thanks V. Uritsky for his help in evaluating this paper.

\section{References}

Falconer, K. J.: Fractal Geometry: Mathematical Foundations and Applications., John Wiley \& Sons, New York, 1995.

Feder, J.: Fractals, Plenum Press, New York, 1988.

Hallinan, T. J. and Davis, T. N.: Small scale auroral arc distortions, Planet. Space Sci., 18, 1735-1744, 1970.

Kozelov, B. V.: Application of methods of fractal analysis to data of ground based observations, in: Equipment and Methods of Geophysical Measurements, edited by Ivanov, V. E., Kola Science Center RAS, Murmansk, 107-118, 1997.

Kozelov, B. V.: Fractal characteristics of spatial structure of aurora, in: Phisics of the near-earth space, Kola Science Centre RAS, Apatity, 572-596, 2000.

Kozelov, B. V., Kozelova, T. V., and Kornilova, T. A.: Dynamics of auroral intensification as an output of magnetosphere-ionosphere system, Proceedings of Sixth International Conference on Substorms, University of Washington, Seattle, Washington, USA, March 25-29, 2002, edited by Winglee, R. M., 432-437, 2002.

Lui, A. T. Y., Chapman, S. C., Liou, K., Newell, P. T., Meng, C. I., Brittnacher, M., and Parks, G. K.: Is the dynamic magnetosphere an avalanching system? Phys. Res. Lett., 27, 7, 911, 2000.

Lyons, L. R., Koskinen, H. E. J., Blake, J. B., Egeland, A., Hirahara, M., Oieroset, M., Sandholt, P. E., and Shiokawa, K.: Processes leading to plasma losses into the high-latitude atmosphere, in: Magnetospheric plasma sources and losses, Space Science Series of ISSI, vol.6, edited by Hultqvist, B., Oieroset, M., Paschmann, G., and Teumann, R., Kluwer, Dordrecht, 85-136, 1999.

Partamies, N., Freeman, M. P., and Kauristie, K.: On the winding of auroral spirals: Interhemispheric observations and Hallinan's theory revisited, J. Geophys. Res., 106, 28 913-28 924, 2001.

Trondsen, T. S. and Cogger, L. L.: A survey of small-scale spatially periodic distortions of auroral forms, J. Geophys. Res., 103, 9405-9415, 1998. 